\title{
Potencialidades Antropométricas físicas-condicionales de Atletas con Discapacidad Intelectual para Olimpiadas Especiales.
}

\section{RESUMEN}

\author{
Daniela Milagros Palacio-González \\ dpalacio@uclv.cu \\ Antonio Jesús Pérez-Sierra \\ antonio.perez@unison.mx
}

Alberto Bautista Sánchez-Oms

asoms@uclv.cu

Yoel Morales-Cuellar

ymorales@uclv.cu

Julio Montes-Brown

julio.montes@unison.mx

Universidad Central "Marta Abreu"

Las Villas-Cuba

Universidad de Sonora-México

El trabajo tiene como objetivo central valorar la condición física en atletas con discapacidad intelectual del Programa de Olimpiadas Especiales en el Atletismo, a partir de indicadores antropométricos físicas-condicionales. Se realizó un estudio descriptivo, exploratorio, no experimental desarrollado bajo un paradigma cualitativo, donde participaron 10 atletas con discapacidad intelectual, integrantes del equipo de Atletismo del Programa de Olimpiadas Especiales en Villa Clara. Se emplearon los métodos el análisis de documentos, la encuesta, la entrevista, la medición, análisis estadístico. Además, se realizaron mediciones antropométricas en miembros superiores e inferiores como alcances, longitudes y circunferencias, así como, indicadores de capacidades físicas condicionales como flexibilidad, fuerza de brazos, abdomen y piernas; rapidez y resistencia. Se utilizó el criterio de inclusión. Los resultados arrojaron las principales características antropométricas físicas-condicionales que distinguen a los atletas, así como, su análisis en función de las potencialidades para el deporte, a partir del comportamiento de los valores medios de cada indicador. La confiabilidad y validez de los instrumentos, nos arrojaron un intervalo de confianza del (95\%), con un valor significación es $(\mathrm{p}<0.05)$.

Palabras clave: Indicadores; discapacidad intelectual; antropométricos; físicas condicionales 


\title{
Anthropometric physical-conditional potentialities of Athletes with intellectual disabilities for Special Olympics
}

\begin{abstract}
The main objective of this work is to evaluate the physical condition of athletes with intellectual disabilities of the Special Olympics Program in Athletics, based on physicalconditional anthropometric indicators. A descriptive, exploratory, non-experimental study was carried out under a qualitative paradigm, with the participation of 10 athletes with intellectual disabilities, members of the Athletics team of the Special Olympics Program in Villa Clara. The methods used were document analysis, survey, interview, measurement, statistical analysis. In addition, anthropometric measurements were taken in upper and lower limbs such as reaches, lengths and circumferences, as well as indicators of conditional physical capacities such as flexibility, strength of arms, abdomen and legs; speed and endurance. Inclusion criteria were used. The results showed the main physical-conditional anthropometric characteristics that distinguish the athletes, as well as their analysis according to the potentialities for the sport, from the behavior of the mean values of each indicator. The reliability and validity of the instruments showed a confidence interval of $95 \%$, with a significance value of $(p<0.05)$.
\end{abstract}

Keywords: Indicators; intellectual disability; anthropometric; conditional physics

Artículo recibido: 10. Junio. 2021 Aceptado para publicación: 16. Julio. 2021 Correspondencia: antonio.perez@unison.mx Conflictos de Interés: Ninguna que declarar 


\section{INTRODUCCIÓN}

Las actividades físicas, el deporte y el ejercicio físico, tienen gran importancia para desarrollar las habilidades y destrezas físicas de las personas con alguna discapacidad, estos constituyen factores excepcionales para potencializar y mejorar la preparación personal, social y psicológico de esta población, a través del deporte adaptado con el propósito de fortalecer y estimular los esfuerzos que ponen en su desempeño este tipo de población, lo cual debemos tomar como ejemplo.

El deporte adaptado son todas las actividades físicas, deportivas, recreativas que pueden hacer o lograr determinada población con necesidades o condiciones para realizar las actividades sociales de participación de los atletas, maximizando sus emociones y sus condiciones físicas. Como expresan Olmos y Urdampilleta (2012, p.1).

Consideramos que un aspecto determinante en estos atletas son sus potencialidades emocionales para poder expresar sus motivaciones a través del deporte adaptado. En los últimos 50 años, estamos viviendo en una sociedad donde aspiramos a la igualdad y equidad para la participación de atletas con discapacidad intelectual en participación a nivel nacional en las Olimpiadas Especiales en el ámbito deportivo, como a escaños internacionales como los Juegos Paralímpicos con el propósito de promover la inclusión de atletas que puedan demostrar todas sus capacidades para contribuir a un cambio de mentalidad de las personas convencionales. (Torralba et al., 2014).

La motivación constituye un factor decisivo en la práctica las actividades físicas, el deporte y el ejercicio físico en las personas con discapacidad, por eso resulta conveniente incentivar de manera emocional y sistemática a estas personas que realizan un esfuerzo extraordinario en su empeño personal para obtener, mejorar y gestionar sus habilidades y capacidades de adaptación para desarrollar su autoestima estilo de vida saludable, como es definido por (Muñoz et al., 2017, p.145).

Por consiguiente, la Federación Cántabra de Deporte para Discapacitados Físicos (2016, p.1) para que el deporte adaptado sea posible también es necesario que se adapten otros aspectos, como los transportes, los edificios o las vías públicas.

Los deportes son adaptados según las necesidades y características de cada disciplina en particular con el propósito de que manifiesten todas sus habilidades y capacidades físicas, técnicas, tácticas y psíquicas, como lo postula Zucchi (2001, p.1). 
De acuerdo a lo expresado, los deportes adaptados ofrecen la equidad e igualdad de oportunidades para las personas con potencialidades físicas-condicionales, para tangán el derecho en el ámbito recreativo y competitivo de expresar sus potencialidades; además, les permiten practicar actividades físicas y deportivas a nivel nacional e internacional.

En el caso del deporte adaptado, sus beneficios guardan una amplia relación con la rehabilitación; a nivel físico permite tener nuevas experiencias de movimiento, facilita el descubrimiento de habilidades, desarrolla capacidades motrices y destrezas propiamente deportivas, potencia el desarrollo funcional y limita las comorbilidades asociadas a la condición primaria de salud; además beneficia a nivel psicosocial el propio autocuidado, capacidad de trabajo, mejora la autoestima y autoeficiencia, y la motivación por un objetivo.

El principal objetivo proporcionar la formación deportiva para la competición atlética durante un ciclo de un año o un cuatrienio de deportes olímpicos a todas las personas mayores de 8 años de edad con discapacidad según las necesidades de personas con cierta discapacidad para desarrollar la aptitud física, demostrar valor, experimentar alegría y participar en un intercambio de dones, destrezas. (Special Olympics Inc., 2009 a).

$\mathrm{Su}$ visión es un movimiento global sin precedente, que, a través de entrenamiento deportivo y competición de calidad, mejora las vidas de las personas con discapacidad intelectual, según las potencialidades físicas-condicionales que las personas con discapacidad intelectual para llegar a ser ciudadanos útiles y productivos, los cuales sean aceptados y respetados en sus comunidades. (Special Olympics Inc., 2009 b).

El número de pruebas o eventos de Atletismo disponibles en Olimpiadas Especiales está orientado para ofrecer oportunidades de competencia a todos los atletas de todas las habilidades y posibilidades. Los entrenadores son responsables de ofrecer entrenamiento especializados y específicos las pruebas o eventos apropiados a cada uno de los atletas dependiendo de la destreza e interés del mismo. El Atletismo de Olimpiadas Especiales incluye eventos de pista, de campo y combinados. En ese sentido los eventos de pista contienen carreras desde los 25 hasta los 10.000 metros; carreras con Vallas de 110 metros para hombres y 100 metros para mujeres; relevos de 4 x 100 metros; 4 x 400 metros y estos mismos eventos con carácter "Unificado", es decir, donde puede incluirse un atleta sin discapacidad intelectual. (Special Olympics Inc., 2009 c). 
Entre los eventos combinados; el pentatlón (100 metros, salto de longitud, impulsión de bala, salto de altura, 400 metros). En los eventos de Marcha Olímpica Asistida 10, 25 y 50 metros; eventos en Silla de Ruedas en carreras de 10, 25, 30, 50 metros; Impulsión de Bala en Silla de Ruedas; carreras de Ruta; medio maratón y maratón.

Cuba se incorpora activamente al movimiento de Olimpiadas Especiales en la década del 80, tanto nacional como internacional donde se destaca el Atletismo como el deporte de mejores y mayores resultados.

Las mediciones antropométricas han sido abordadas por autores como (Gómez et al., 2002; De la Vega et al., 2004; Vasques et al., 2010; Pérez et al., 2013; Carménate et al., 2014; Cossio et al., 2014; Nikic et al., 2017; Castillo y Espinoza, 2018). Con relación a las mediciones antropométricas en personas con discapacidad intelectual, estos últimos enfatizan en que, a los escolares con discapacidad intelectual, se debían caracterizar antropométricamente (.....), deberían ser atendidos y estudiados desde varios puntos de vista, con el propósito de proporcionar información respecto al estado de salud general de esta población.

Según Palacio (s.f., 6). Afirma que "es importante que el profesor llegue a conocer y determinar el perfil personal del niño con necesidades, a fin de poder establecer con exactitud el tipo de adaptación"

En el contexto deportivo el Programa Nacional Olimpiadas Especiales en Cuba ha alcanzado niveles relevantes en cuanto a organización desde la base, incorporación y preparación de atletas, capacitación de entrenadores, voluntarios y familias; inclusión de nuevos deportes; en este contexto.

En el contexto deportivo el Programa Nacional Olimpiadas Especiales en Cuba ha alcanzado niveles relevantes en cuanto a organización desde la base, incorporación y preparación de atletas, capacitación de entrenadores, voluntarios y familias; inclusión de nuevos deportes; en este contexto.

El deporte de Atletismo constituye una fortaleza en el programa desde la base hasta los resultados internacionales del programa, sin embargo, en la práctica del Programa provincial de Olimpiadas Especiales en la provincia de Villa Clara, aun cuando, se han alcanzado resultados favorables en las competiciones, se ha desarrollado el entrenamiento de este deporte con un carácter asistemático y empírico, pues no se ha partido de un 
conocimiento real del estado de indicadores básicos de la condición física, que pudieran favorecer el rendimiento en la práctica del Atletismo.

En ese sentido, subsiste la situación problemática relacionada con el desconocimiento de indicadores antropométricos y funcionales de atletas con discapacidad intelectual para su incorporación a la práctica deportiva de Olimpiadas Especiales, específicamente el Atletismo; por lo que esta investigación tiene como objetivo, valorar la condición física en atletas con discapacidad intelectual del Programa de Olimpiadas Especiales en el Atletismo, a partir de indicadores antropométricos físicas-condicionales.

\section{ESTRATEGIAS METODOLÓGICAS O MATERIALES Y MÉTODOS}

Se realizó una investigación cualitativa sustentada en estudio descriptivo, exploratorio, no experimental, donde participaron 10 atletas con discapacidad intelectual, integrantes del equipo de Atletismo del Programa de Olimpiadas Especiales en Villa Clara En la investigación utilizamos los siguientes métodos de nivel empírico como se describen a continuación.

El análisis de documentos: La Estrategia de Desarrollo del Subprograma de Olimpiadas Especiales en la provincia de Vila Clara, al plan de entrenamiento de los entrenadores de atletas que asisten a Olimpiadas Especiales, al Expediente del Centro de Diagnóstico y Orientación (CDO), de cada atleta; todo con el propósito de constatar en la documentación oficial, aspectos relacionados con la evaluación antropométrica.

La encuesta: Se les aplico a entrenadores del Subprograma Provincial de Olimpiadas Especiales, específicamente los del deporte Atletismo, con el objetivo de constatar la existencia de mediciones anteriores.

La entrevista: Fue destinada para los funcionarios del Subprograma Provincial de Olimpiadas Especiales, específicamente los del deporte Atletismo; para conocer las orientaciones emitidas con respecto a evaluaciones antropométricas en estos atletas.

La medición: A los atletas, para medir indicadores antropométricos de los atletas, alcances y longitudes de las extremidades superiores e inferiores. Según Ruiz et al. (2009, p.4).

Análisis estadístico: Se empleó con el propósito de determinar los datos obtenidos con el programa estadístico SPSS versión 23, aplicando los estadígrafos la media en los indicadores antropométricos del tren superior e inferior y los indicadores funcionales, el procesamiento estadístico la media aritmética y la frecuencia, para constatar los valores 
medios y la posición de los atletas con respecto a estos. La confiabilidad y validez de los instrumentos, nos arrojaron un intervalo de confianza del (95\%), con un valor significación es $(\mathrm{p}<0.05)$.

Además, se utilizó el criterio de inclusión de los padres de familias de los 10 atletas para sus consentimientos y directivos.

\section{Población}

La población la conforman los diez escolares atletas que integran el equipo masculino de Atletismo de Olimpiadas Especiales en la Provincia de Villa Clara, categoría 13-15 años, todos varones. Los mismos pertenecen a diferentes escuelas especiales para la educación de escolares con discapacidad intelectual. Residen en diferentes municipios de la provincia, a la que han representado en distintas competiciones nacionales.

Tabla 1: Caracterización de la población de atletas

\begin{tabular}{crrll}
\hline Cantidad de atletas & Sexo & \multicolumn{1}{l}{ Edad } & \multicolumn{1}{l}{ Peso } & \multicolumn{1}{l}{ Talla } \\
\hline 10 & $\mathrm{M}$ & $\pm 13,6$ & $\pm 58,3$ & $\pm 1,605$ \\
\hline
\end{tabular}

\section{Procedimientos}

El proceso de investigación se desarrolló bajo las normas y principios éticos expresados en la Declaración de Helsinki 2013. Antes de comenzar la sesión de entrenamiento, con vestimenta adecuada los especialistas en medicina deportiva realizaron las mediciones antropométricas los del tren superior e inferior, estas mediciones se tomaron tres veces para comprobar el grado de diferencia de entre ellas, con un nivel de confianza de $95 \%$. El laboratorio donde se aplicó estas mediciones cumple con las normas estandarizadas de certificación de la Sociedad Internacional para el avance de la Cineantropometría (ISAK) nivel II.

Los indicadores funcionales de las físicas-condicionales, posterior a un calentamiento articular previamente realizado en la pista de atletismo se realizaron de manera ordenada como se expresan a continuación: Flexibilidad, Rapidez, Planchas, Abdominales, Salto largo sin impulso y Resistencia todas estas pruebas fueron realizadas el mismo día.

\section{Instrumentos}

Para la confiabilidad y validez de los instrumentos se seleccionaron dos evaluadores certificados ISAK de nivel II del Departamento Medicina del Deporte, los cuales poseen toda la credibilidad de conocimientos y experiencia. 
Se utilizó cinta métrica marca SURTEK CL 20 (66), medidas en metros y pulgadas con el propósito de medir los indicadores diámetros, longitud y los alcances antropométricos de los sujetos motivos de investigación.

Cronómetro Manual marca JIMHER STOP WATCH SG-152, este instrumento nos facilitó tomar los tiempos de los diez escolares atletas que integran el equipo masculino de Atletismo de Olimpiadas Especiales de las capacidades físicas-condicionales.

Planilla de Registro de Datos, diseñado el programa Excel, este instrumento fue de gran utilidad práctica para registrar los datos de las pruebas antropométricas físicascondicionales; además este registró datos nos permitió aplicar a través del programa SPSS versión 23 para los indicadores estadísticos.

\section{RESULTADOS Y DISCUSIÓN}

Tabla 2: Resultados frecuencias de atletas que sobrepasan la media de las mediciones de los indicadores antropométricos del tren superior.

\begin{tabular}{lccccccc}
\hline \multicolumn{1}{c}{ Indicador } & Media & $\begin{array}{c}\text { Atletas por } \\
\text { encima de la } \\
\text { media }\end{array}$ & $\%$ & $\begin{array}{c}\text { Atletas por } \\
\text { debajo de } \\
\text { la media }\end{array}$ & $\begin{array}{c}\text { Atletas } \\
\text { que } \\
\text { igualan }\end{array}$ & $\%$ \\
\hline $\begin{array}{l}\text { Alcance hacia arriba } \\
\text { (desde piso }\end{array}$ & 1.97 & 4 & 40 & 5 & 50 & 0 & 0 \\
$\begin{array}{l}\text { Alcance hacia delante } \\
\text { Alcance Lateral }\end{array}$ & 0.73 & 6 & 60 & 4 & 40 & 0 & 0 \\
$\begin{array}{l}\text { Longitud antebrazo } \\
\text { Longitud de la mano }\end{array}$ & 0.67 & 4 & 40 & 6 & 60 & 0 & 0 \\
$\begin{array}{l}\text { Longitud de la palma } \\
\text { de la mano }\end{array}$ & 0.16 & 4 & 30 & 7 & 70 & 0 & 0 \\
$\begin{array}{l}\text { Ancho de la palma de la } \\
\text { mano }\end{array}$ & 0.8 & 3 & 50 & 4 & 40 & 2 & 20 \\
\hline
\end{tabular}

$\overline{\text { Se aprecia en la tabla que los indicadores donde los atletas se encuentran por encima de }}$ la media y en la media. están: alcance hacia delante (6 atletas) y longitud de la palma de la mano (5 atletas). Estos atletas tienen potencialidades para las pruebas de lanzamiento pues la longitud del brazo incide en la altura inicial de vuelo del lanzamiento. el ángulo inicial de vuelo y el recorrido de aplicación de fuerza. que son factores determinantes de los resultados en estas pruebas. En el caso de la longitud de la palma de la mano también incide favorable en el agarre del implemento. lo cual le brinda una mayor y segura sujeción del mismo. 
Tabla 3: Resultados frecuencias de atletas que sobrepasan la media en los indicadores antropométricos del tren inferior.

\begin{tabular}{lccccccc}
\hline & Media & $\begin{array}{c}\text { Atletas } \\
\text { por } \\
\text { encima } \\
\text { de la } \\
\text { media }\end{array}$ & $\begin{array}{c}\text { Atletas } \\
\text { por } \\
\text { debajo } \\
\text { de la } \\
\text { media }\end{array}$ & $\begin{array}{c}\text { Atletas } \\
\text { que } \\
\text { igualan }\end{array}$ & $\%$ \\
\hline Longitud cadera-piso & 0.89 & 2 & 20 & 8 & 80 & 0 & 0 \\
Longitud cadera-rodilla & 0.43 & 2 & 20 & 6 & 60 & 0 & 0 \\
Longitud rodilla-tobillo & 0.4 & 3 & 30 & 5 & 50 & 1 & 10 \\
Longitud del pie & 0.25 & 2 & 20 & 6 & 60 & 2 & 20 \\
Circunferencia Muslo & 0.4 & 4 & 40 & 5 & 50 & 1 & 10 \\
Circunferencia Pantorrilla & 0.32 & 5 & 50 & 4 & 40 & 1 & 10 \\
\hline
\end{tabular}

Se puede observar en esta tabla. que no hay atletas que sobrepasan la media. sin embargo. hay atletas que se encuentran en la media en el indicador circunferencia de pantorrilla (5 atletas) lo cual puede incidir en la masa necesaria para producir fuerza y cercano a la media. el indicador circunferencia del muslo donde cuatro atletas presentan condiciones para también generar fuerza mediante la contracción muscular. Estos atletas tienen potencialidades para logra resultados competitivos en las pruebas de saltos. lanzamientos y velocidad.

Tabla 4 Resultados frecuencias de atletas que sobrepasan la media en los indicadores funcionales.

\begin{tabular}{lcccccc}
\hline Total & $\begin{array}{c}\text { Por encima } \\
\text { de la media }\end{array}$ & $\%$ & $\begin{array}{c}\text { Por debajo } \\
\text { de la media }\end{array}$ & $\%$ & Igualan & $\%$ \\
\hline Flexibilidad & 6 & 60 & 4 & 40 & 0 & 0 \\
Rapidez & 5 & 50 & 4 & 40 & 1 & 10 \\
$\begin{array}{l}\text { Planchas } \\
\text { Abdominales }\end{array}$ & 4 & 40 & 6 & 60 & 0 & 0 \\
$\begin{array}{l}\text { Salto largo Sin } \\
\text { carrera de impulso }\end{array}$ & 5 & 50 & 5 & 50 & 0 & 0 \\
Resistencia & 4 & 50 & 5 & 50 & 0 & 0 \\
\hline
\end{tabular}


Con relación a los indicadores funcionales es importante destacar que en la flexibilidad hay 6 atletas por encima de la media y 5 atletas que están en la media en indicadores tales como: rapidez abdominal y salto de longitud sin carrera de impulso lo cual refleja que a pesar de que en tablas anteriores algunos atletas tienen posibilidades de lograr buenos resultados en pruebas de lanzamiento saltos y velocidad, lo cierto es que la mitad o más de los atletas. No están en condiciones de mostrar buenos resultados en dichas pruebas debido a que están por debajo de la media lo cual puede estar influenciado por la edad pues la fuerza sé sensible posterior a los 15 años influyendo está en las pruebas de velocidad.

En el caso de las disciplinas o pruebas de resistencia no se aprecian atletas con condiciones para un rendimiento en pruebas de resistencia por lo que los entrenadores deben enfatizar en la preparación de los atletas con insuficiente desarrollo y potenciar la condición física de los que presentan una adecuada preparación.

Es necesario que los entrenadores conozcan las características individuales de sus atletas como también sus fortalezas oportunidades, debilidades y amenazas, relacionados con los fundamentos biológicos, físicos y psicológicos para la planificación entrenamiento deportivo. Según las potencialidades antropométricas físicas-condicionales de atletas con discapacidad intelectual.

\section{CONCLUSIÓN O CONSIDERACIONES FINALES}

Los resultados de los indicadores analizados muestran que hay 5 de los 10 atletas que presentan potencialidades para lograr buenos resultados en las Olimpiadas Especiales. por lo que se les debe prestar atención diferenciada y continuar preparando a aquellos que no han mostrado una buena condición física para alcanzar resultados destacados en dichas olimpiadas.

\section{LISTA DE REFERENCIAS}

Aguado. I. N.; y Mencho. I. (2007). La participación camagüeyana en Olimpiadas Especiales. Revista PODIUM, 2(1), 82-87.

http://podium.upr.edu.cu/index.php/podium/article/view/33

Canda. A. S. (2012). Variables antropométricas de la población deportista española. Editorial. Consejo Superior de Deportes. https://munideporte.org/imagenes/documentacion/ficheros/029C0791.pdf

Carménate. L.; Moncada. F. A.; y Borjas. E. W. (2014). Manual de medidas 
antropométricas. Editorial. SALTRA.

https://repositorio.una.ac.cr/bitstream/handle/11056/8632/MANUAL\%20ANTR OPOMETRIA.pdf?sequence $=1 \&$ isAllowed $=\mathrm{y}$

Castillo. N.; y Espinoza. C. (2018). Condición Física y medidas Antropométricas de estudiantes continentales: una comparación con estudiantes insulares del archipiélago Juan Fernández de la región de Valparaíso. Revista Horizonte. Ciencias de la Actividad Fisica y salud, 9(1), 40-50.

https://minio2.123dok.com/dt02pdf/123dok_es/original/2020/11_19/bgnfpk1605 $\underline{758576}$

Cossio. M.; Vidal. R.; Lagos. J.; y Gómez. R. (2015). Perfil antropométrico en función del estado nutricional de niños con discapacidad intelectual. Revista chilena de pediatría, 86(1),18-24. https://dx.doi.org/10.1016/j.rchipe.2015.04.004

De la Vega Bustillos. E. J.; López. F. O.; y Soto. S. (2004. 26 al 29 de mayo). Antropometría para discapacitados. [conferencia]. VI Congreso Internacional de Ergonomía. Guanajuato. Mexico. http://www.semac.org.mx/archivos/6-22.pdf

España. Federación Cántabra de Deporte para Discapacitados Físicos (2016). Deporte y Superación. http://www.fcddf.org/deporte-y-superacion/

Gómez. S.; Agudelo. A.; García. Y.; y Franco. K. (2020). Correlación del índice de masa corporal con diferentes indicadores antropométricos en estudiantes de una universidad privada en Medellín Colombia periodo 2016-2018. Revista J. Health Med. sci.,6(3),177-183.

http://www.johamsc.com/wp-content/uploads/2020/09/JOHAMSC-6.3-177-183GARCIA-013-20-2020.pdf

Maldonado. A.; Romero. R.; Zapata. J. J.; Martínez. E.; y Noriega. S. (2015). Desarrollo de datos antropométricos para niños con discapacidad motriz en ciudad Juárez. Revista Cultura Científica Y Tecnológica, (41), 145-153.

http://erevistas31.uacj.mx/ojs/index.php/culcyt/article/view/285

Mónaco. International Amateur Athletic Federation (2009 a, b, c). Special Olympics. Reglamentos Generales Oficiales. Editorial. Special Olympics. https://media.specialolympics.org/resources/leading-a-program/generalrules/General-Rules-Spanish.pdf 
Mónaco. International Amateur Athletic Federation (2009). 9 Annual Report. Leaving a Legacy For the future. Editorial. Special Olympics Be a Fan.

https://media.specialolympics.org/resources/reports/annualreports/2009AnnualReport.pdf

Muñoz. E. M.; Garrote. D.; y Sánchez. C. (2017). La práctica deportiva en personas con discapacidad: motivación personal. inclusión y salud. International Journal of Developmental and Educational Psychology INFAD Revista de Psicología, 4(1), 145-152. https://doi.org/10.17060/ijodaep.2017.n1.v4.1037 https://www.redalyc.org/pdf/3498/349853537015.pdf

Nikic. M. (2017). Valoración fisiológica y antropométrica de las personas con discapacidad visual que practican deporte en España. [Tesis Doctoral, Universitat de Barcelona]. http://diposit.ub.edu/dspace/bitstream/2445/131510/1/MNZ_TESIS.pdf

Olmos. J.; y Urdampilleta. A. (2012). El deporte adaptado en la edad escolar: criterios de inclusión e integración social. Revista EFDeporte, (172), 1-1. https://www.efdeportes.com/efd172/el-deporte-adaptado-en-la-edad-escolar.htm

Palacio. D. M. (s.f.). Las adaptaciones de las tareas motrices de la Educación Física; una necesidad para la inclusión de escolares con limitaciones físico motoras. https://www.researchgate.net/profile/Daniela-Palacio-

Gonzalez/publication/331208194_Las_adaptaciones_de_las_tareas_motrices_de _la_Educacion_Fisica/links/5c6c3479299bf1e3a5b62a7a/Las-adaptaciones-delas-tareas-motrices-de-la-Educacion-Fisica.pdf

Pérez. J.; Ocete. C.; Soto. J.; Ossa. A.; García. J.; M.; y García. J. (2013). Centro de Estudios sobre Deporte Inclusivo: Tres años de fomento del deporte inclusivo a nivel práctico. académico y científico. Editorial. Universidad Politécnica de Madrid. Fundación Sanitas.

https://www.researchgate.net/publication/320345827_Centro_de_Estudios_sobr e_Deporte_Inclusivo_Tres_anos_de_fomento_del_deporte_inclusivo_a_nivel_pr actico_academico_y_cientifico

Ruiz. G. A.; Ochoa. A.; de la Vega. E. J.; y Villarreal. C. L. (2009). La antropometría en el desarrollo de nuevos productos. (Ponencia). XV Congreso Internacional de Ergonomía SEMAC. Ciudad de México. México. 
http://www.semac.org.mx/archivos/congreso11/ANTRO4.pdf

Torralba. M. A.; Braz. M.; y Rubio. M.; J. (2014). Motivación en el deporte adaptado. Revista Psychology. Society. \& Education, 6(1),27-40. doi: http://dx.doi.org/10.25115/psye.v6i1.506 http://ojs.ual.es/ojs/index.php/psye/issue/view/59

Vasques. A. C.; Priore. S. E.; Frandsen. L. E.; y Franceschini, S. (2010). Uso de medidas antropométricas para evaluar la acumulación de grasa visceral. Revista de Nutrição, 23(1), 107-118. https://doi.org/10.1590/S1415-52732010000100012

Zucchi. D. G. (2001). Deporte y discapacidad. Revista EFdeportes, (43), 1-2. https://www.efdeportes.com/efd43/discap.htm 